\title{
Puesta al día
}

\section{ESTOMATITIS ASOCIADA A INHIBIDORES mTOR: PUESTA AL DÍA EN LA VALORACIÓN, PREVENCIÓN Y MANEJO.}

mTOR INHIBITOR-ASSOCIATED STOMATITIS: AN UPDATE IN THE ASSESSMENT, PREVENTION AND MANAGEMENT

\author{
Juan José Arévalo Manso ${ }^{1,2}$,juan lgnacio Navarro Ruiz', Ma Consuelo Arévalo de \\ Frutos', Susana Hernández Sánchez', Mạ Jesús Álvaro Miguel', Raúl Coto Linde' \\ 'Unidad de Oncología y Hematología. Complejo Asistencial de Segovia. \\ ${ }^{2}$ Servicio de Asesoría en Investigación Sanitaria. CSIF-Sanidad. Segovia.
}

Autor para correspondencia:

Juan José Arévalo Manso. Correo electrónico:jarevaloes@hotmail.com

\begin{abstract}
RESUMEN
Introducción: Estomatitis y mucositis son términos utilizados como sinónimos a menudo, pero son procesos con características diferenciadoras. La correcta identificación y manejo de la estomatitis asociada a la administración de inhibidores mTOR (EAIm) favorecería una mejor evolución del paciente. El cuerpo de conocimiento de la EAIm es limitado, y casi inexistente la literatura en español.
\end{abstract}

Objetivo: Realizar una puesta al día sobre la valoración, prevención y manejo de la EAIm.

Método: Revisión no sistemática en las bases de datos PubMed, IBECS y Cuiden, utilizando los descriptores "Stomatitis", "TOR Serine-Threonine

Kinases/antagonists and inhibitors" y "Practice Guideline [Publication Type]".

Resultados: Se encontraron un total de 2 guías clínicas y 12 artículos de revisión con recomendaciones específicas 
respecto a la valoración, prevención y manejo de la EAIm.

Conclusión: La EAIm es un evento adverso que puede ser confundido con la mucositis asociada a la quimioterapia convencional. Son procesos diferentes, que requieren abordajes diferentes. El manejo adecuado de la EAIm propiciaría una mejora de la calidad de vida del paciente, la resolución temprana de las lesiones y la continuidad del tratamiento. No obstante, la EAIm no cuenta con un cuerpo de conocimiento importante respecto a su valoración, prevención y manejo, lo que tiene implicaciones importantes para la investigación en los próximos años.

Palabras clave (DeCS): Estomatitis; Serina-Treonina Quinasas TOR/antagonistas e inhibidores; Efectos colaterales y reacciones adversas relacionadas con medicamentos; Evaluación de síntomas; Revisión; Enfermería basada en la evidencia.

\section{ABSTRACT}

Introduction: Stomatitis and mucositis usually are used as synonyms, but they have differentiating features. A correct diagnosis and management of mTOR inhibitors-associated stomatitis (mIAS) could improve the patient's outcomes. mIAS body of knowledge is limited, and nearly non-existent in spanish literature.

Aim: To do a knowledge update about the assessment, prevention and management of mIAS. Method: Non-systematic literature review in PubMED, IBECS and Cuiden databases, by using the MeSH terms "Stomatitis", "TOR SerineThreonine Kinases/antagonists and inhibitors" and "Practice Guideline [Publication Type]".

Results: A total of 2 clinical practice guidelines and 12 review papers with specific recommendations on mIAS were found.

Conclusion: mIAS is a side effect that can be mistaken to chemotherapy-associated

mucositis. They are different outcomes entailing for a different approach. A right managenet of mIAS could improve patient's quality of life, an earlier healing and the continuity of treatment. However, mIAS has a limited body of knowledge in literature. This has major research implications for the future.

Keywords (MeSH): Stomatitis; TOR Serine-Threonine Kinases/antagonists and inhibitors; Drug-related side effects and adverse reactions; Symptom assessment; Review; Evidence-based nursing. 


\section{INTRODUCCIÓN}

Los inhibidores mTOR (everolimus, temsirolimus, ridaforolimus, sirolimus) son un grupo de fármacos utilizados como terapia dirigida en el tratamiento de varios tipos de cáncer y como inmunosupresores para evitar el rechazo en pacientes transplantados.

Su uso es relativamente reciente, por lo que el conocimiento sobre la prevención y el manejo de sus efectos adversos es más limitado en comparación con los quimioterápicos convencionales. Entre los efectos adversos de los inhibidores mTOR se incluyen astenia, hiperglucemia, hiperlipidemia, hipofosfatemia, toxicidad hematológica, náuseas, diarrea 0 problemas mucocutáneos, siendo la estomatitis el más común de todos ellos ${ }^{1,2}$.

Estomatitis y mucositis son términos que a menudo se confunden y son utilizados como sinónimos, pero la estomatitis asociada al tratamiento con inhibidores mTOR (EAIm) presenta diferencias con la mucositis que hacen que sean considerados procesos diferenciados ${ }^{3,4}$. Los datos relativos a la valoración, prevención y manejo de la EAIm no son muy abundantes en la literatura a nivel global, y las publicaciones en español son prácticamente inexistentes.

El objetivo de este artículo es mostrar las diferencias entre ambas entidades clínicas y realizar una puesta al día sobre la valoración, prevención y manejo de la EAIm mediante una revisión (no sistemática) de las recomendaciones facilitadas por las principales guías clínicas y otros artículos de revisión.

\section{MÉTODO}

Se realizó una revisión no sistemática en las bases de datos PubMed, IBECS y Cuiden, utilizando los descriptores "Stomatitis", "TOR SerineThreonine Kinases/antagonists and inhibitors" y "Practice Guideline [Publication Type]" o "Review [Publication Type]". En las bases de datos IBECS y Cuiden se utilizaron los Descriptores en Ciencias de la Salud (DeCS) equivalentes. Se tuvieron en cuenta los documentos publicados a partir del año 2000, y se seleccionó la version más actual de cada guía clínica.

Los documentos a seleccionar debían contener recomendaciones relativas a la valoración, prevención y/o manejo específico de la EAIm, siendo excluidos aquellos que únicamente lo hacen refiriéndose a la mucositis oral asociada a la administración de quimioterapia. Para esta revision se tuvieron especialmente en cuenta aquellas recomendaciones que contaban con mayor grado de consenso entre los artículos seleccionados.

Utilizando los documentos seleccionados, se prentendió dar respuesta a cuatro preguntas: (1) ¿Que características diferencian la EAIm de la mucositis asociada a quimitoterapia convencional?, (2) ¿Cómo debe valorarse la EAIm?, (3) ¿Qué medidas de prevención existen?, y (4) ¿Cuál debe ser el manejo y tratamiento de la EAIm?

\section{RESULTADOS}

En total se encontraron 2 guías clínicas y 12 artículos de revisión en los que se hacían recomendaciones específicas relativas a la valoración, 
prevención y/o manejo de la

EAIm.

\section{DIFERENCIA ESTOMATITIS- MUCOSITIS}

La mucositis está asociada a la administración de quimioterápicos convencionales y/o radioterapia en la zona cabeza-cuello. Consiste en una inflamación de la mucosa de la boca, faringe, laringe 0 tracto digestivo. Se manifiesta mediante eritema o ulceraciones en la mucosa, que suelen tener forma irregular o difusa, con un tamaño que puede alcanzar varios centímetros. Las úlceras pueden presentar (o no) eritema perilesional, el cual no suele tener borde elevado, y con frecuencia están recubiertas por una pseudomembrana ${ }^{5}$. Debido a que son dolorosas, su tamaño y su persistencia, suelen afectar a la ingesta oral de los pacientes de forma más importante que en el caso de la EAIm. En ambos casos las lesiones suelen aparecer con mayor frecuencia en las zonas no queratinizadas de la boca, como la mucosa, las partes inferior $y$ lateral de la lengua, el suelo de la boca o el paladar blando.

En cambio, la EAIm presenta algunas diferencias en cuanto a signos, síntomas y ocurrencia en el tiempo, y morfológicamente tienen más parecido con las lesiones de la estomatitis aftosa ${ }^{6}$. Se caracteriza por una o varias ulceraciones superficiales de forma ovoide, espaciadas, de menor tamaño que las lesiones de la mucositis (generalmente menores de $1 \mathrm{~cm}$ ), con una membrana central blanquecina o grisácea rodeada por un anillo eritematoso elevado (Figura 1) ${ }^{7}$. En la mayoría de los casos afecta a la mucosa bucal, aunque también pueden verse afectadas la zona faríngea y esofágica. Para mayor claridad, presentamos una tabla con las diferencias morfológicas $y$ sintomáticas entre estomatitis $y$ mucositis (Tabla 1).

\begin{tabular}{|c|c|c|}
\hline & Estomatitis & Mucositis \\
\hline Forma & $\begin{array}{l}\text { Ovoide o } \\
\text { circular }\end{array}$ & Irregular \\
\hline Tamaño & $\begin{array}{l}\text { Pequeño } \\
(<1 \mathrm{~cm})\end{array}$ & $\begin{array}{l}\text { Variable } \\
(>1 \mathrm{~cm})\end{array}$ \\
\hline Localización & $\begin{array}{c}\text { Zonas no } \\
\text { queratiniza- } \\
\text { das de la } \\
\text { boca, faringe } \\
\text { y/o esófago. }\end{array}$ & $\begin{array}{c}\text { Zonas no } \\
\text { queratiniza- } \\
\text { das de la } \\
\text { boca, } \\
\text { faringe, } \\
\text { laringe, } \\
\text { resto del } \\
\text { tubo } \\
\text { digestivo } \\
\text { (esófago, } \\
\text { intestino) } \\
\end{array}$ \\
\hline $\begin{array}{l}\text { Eritema } \\
\text { perilesional }\end{array}$ & $\begin{array}{l}\text { Si, con } \\
\text { borde } \\
\text { elevado. }\end{array}$ & $\begin{array}{c}\text { Puede } \\
\text { presentarlo, } \\
\text { con o sin } \\
\text { borde } \\
\text { elevado. }\end{array}$ \\
\hline $\begin{array}{l}\text { Superposici } \\
\text { ón de } \\
\text { lesiones }\end{array}$ & $\begin{array}{c}\text { No (lesiones } \\
\text { separadas) }\end{array}$ & $\begin{array}{c}\text { Puede } \\
\text { presentarla }\end{array}$ \\
\hline Membrana & $\begin{array}{c}\mathrm{Si}, \\
\text { blanqueci-na } \\
\text { o grisácea }\end{array}$ & $\begin{array}{c}\text { Puede } \\
\text { presentarla }\end{array}$ \\
\hline Dolor & $\begin{array}{l}\text { Elevado, en } \\
\text { relación con } \\
\text { el tamaño de } \\
\text { las lesiones }\end{array}$ & $\begin{array}{c}\mathrm{Si}, \\
\text { generalmen- } \\
\text { te } \\
\text { proporcional } \\
\text { al tamaño } \\
\text { de las } \\
\text { lesiones. } \\
\end{array}$ \\
\hline
\end{tabular}

Tabla 1. Diferencias morfológicas y sintomáticas entre las lesiones de estomatitis asociada a quimioterapia y/o radioterapia, y lesiones de estomatitis asociada al tratamiento con inhibidores mTOR. Se describen las características que en cada caso se presentan de forma más habitual, pudiendo ocurrir casos que supongan excepciones ${ }^{5-8}$.

Generalmente el inicio de los síntomas de la estomatitis ocurre poco tiempo después de la primera administración del fármaco, normalmente entre 4 y 25 días (10 días de media). 


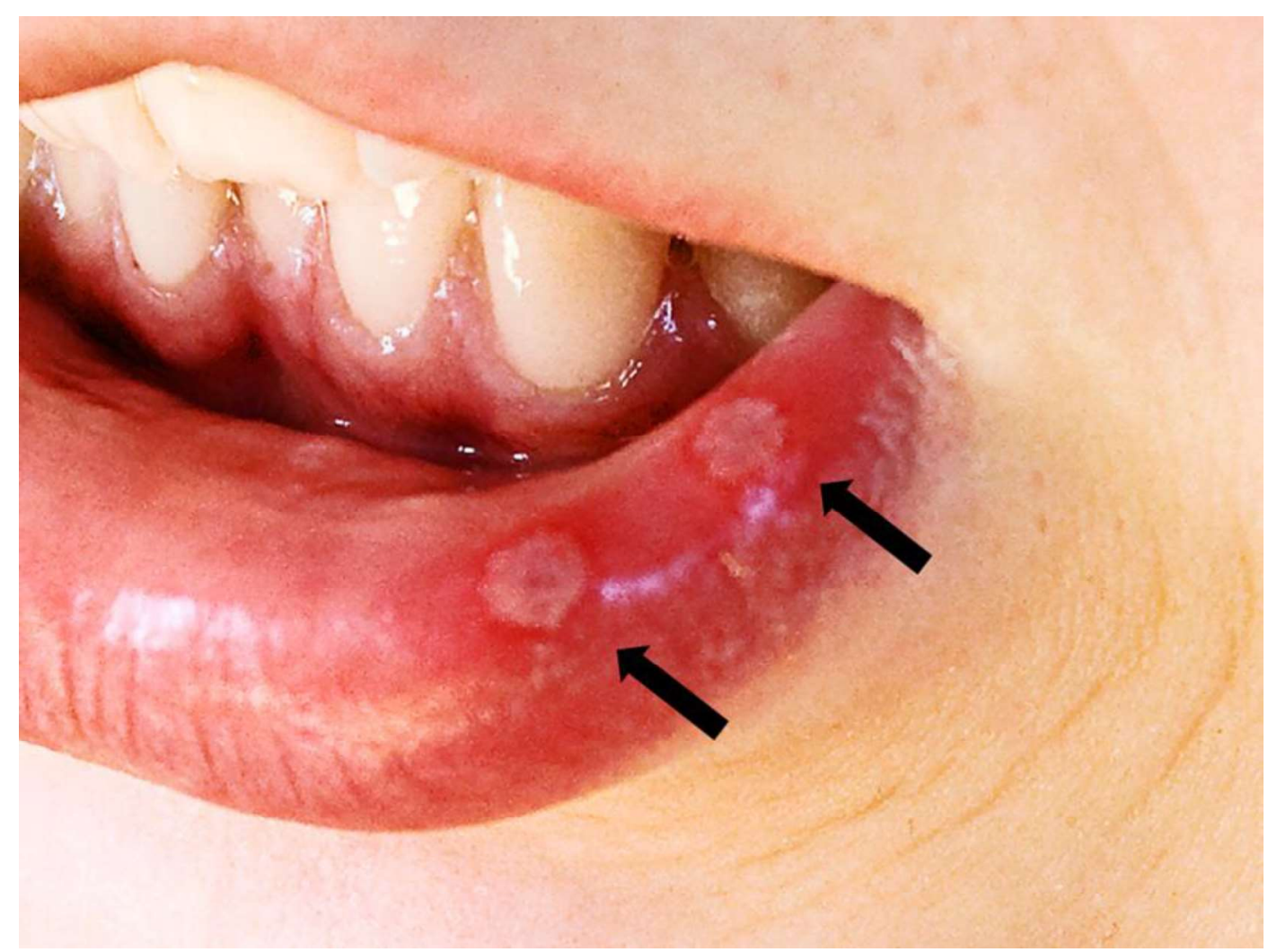

Figura 1. Lesiones ulcerativas en la estomatitis asociada al tratamiento con inhibidores mTOR (EAIm).

Las lesiones suelen ser muy dolorosas con relación a su pequeño tamaño, con una intensidad medida del dolor referido por el paciente de 7 en una escala de $10^{8}$.

La probabilidad de aparición de nuevos episodios parece disminuir a medida que se suman ciclos de tratamiento (tasa de recurrencia del $25 \%$ ), y en caso de ocurrir la gravedad de las lesiones suele ser menor ${ }^{1,9}$.

Los estudios sobre los factores de riesgo asociados al desarrollo de EAIm son muy escasos, pero hasta ahora parece que el tipo de fármaco, la dosis o el tipo de tumor indican en cierta medida qué pacientes tienen más probabilidad de desarrollar las formas más graves de esta complicación. Así los pacientes con tumores neuroendocrinos, gástricos, de pulmón o astrocitomas parecen ser más propensos, así como aquellos tratados con everolimus (el inhibidor mTOR más utilizado en nuestro medio), en comparación con temsirolimus ${ }^{10}$.

La EAIm es la complicación más frecuente en el tratamiento con inhibidores $\mathrm{mTOR}$, representando un $73,4 \%$ de todos los eventos adversos y afectando hasta al $78 \%$ de los pacientes $1,5,7$. Aunque las lesiones suelen desaparecer de forma espontánea en el plazo de dos semanas y ser de carácter leve o moderado, entre un $5 \%$ y un $9 \%$ presenta una afectación de carácter grave que puede 
prolongarse en el tiempo y tener implicaciones importantes. Por un lado, las lesiones son muy dolorosas, lo que deteriora la calidad de vida del paciente $y$ puede condicionar la ingesta oral e incluso el habla ${ }^{11}$.

Por otro, puede limitar el tratamiento oncológico e influir en el pronóstico del paciente, ya que la EAIm motiva el $27,3 \%$ de las reducciones de dosis y el $13,1 \%$ de las interrupciones del tratamiento con inhibidores mTOR ${ }^{1}$. Por lo tanto, aunque EAIm y mucositis son procesos diferenciados, también presentan similitudes que pueden llevar a confundirlas. El personal sanitario que atiende a estos pacientes, y en especial las enfermeras de las unidades de oncología, tiene un papel relevante en la correcta identificación de casos y el manejo correcto de los mismos.

\section{VALORACIÓN}

La EAIm es un indicador de la toxicidad asociada al tratamiento con inhibidores mTOR. Su aparición y gravedad puede condicionar decisiones en cuanto a reducciones de dosis o interrupción del tratamiento oncológico. Por ello la utilización de un método de valoración adecuado es importante para tomar las decisiones correctas en el tratamiento y el cuidado del paciente.

Existen escalas utilizadas para la valoración de la mucositis, pero varios autores coinciden en que éstas no resultan adecuadas para los pacientes con EAIm por presentar limitaciones para valorarla correctamente ${ }^{3,12}$. Al no ser creadas para valorar ese tipo de lesiones y síntomas, este tipo de escalas tienen menor sensibilidad para la detección de la EAIm.

La puntuación en esas escalas depende principalmente del tamaño y la extensión de las lesiones (escalas OMAS y NCICTCAE) o del grado en el que afectan a la ingesta oral (escala de mucositis de la OMS). En cambio, los aspectos principales a la hora de valorar la EAIm son el dolor y la longevidad de las lesiones. Ya que son de pequeño tamaño y pueden ser muy dolorosas, el uso de instrumentos de valoración no específicos puede llevar a una infraestimación de su incidencia y de su gravedad.

En 2013 Boers-Doets y Lalla propusieron una escala específica para valorar adecuadamente la EAIm, la mIAS Scale [13]. El uso de esta escala es fácil y rápido, y está contemplado en las principales guías clínicas ${ }^{12}$. La mIAS Scale consta de dos componentes: uno objetivo (presencia de lesiones y su duración) y otro subjetivo (dolor asociado a las lesiones). Estos componentes se valoran en 4 posibles grados (de 0 a 3 ), según muestra la Tabla 2.

Los autores sugieren que sólo debería considerarse una reducción de la dosis o interrupción del tratamiento en aquellos pacientes con un grado 3 en ambos componentes. 


\begin{tabular}{|l|l|}
\hline & Componente subjetivo \\
\hline Grado 0 & $\begin{array}{l}\text { Sin dolor orofaríngeo atribuido a } \\
\text { EAIm. }\end{array}$ \\
\hline Grado 1 & $\begin{array}{l}\text { Dolor orofaríngeo atribuido a } \\
\text { EAIm, con puntuación media } \leq 2 \\
\text { en una escala de 0-10, en las } \\
\text { últimas 24 horas. }\end{array}$ \\
\hline Grado 2 & $\begin{array}{l}\text { Dolor orofaríngeo atribuido a } \\
\text { EAIm, con puntuación media } \leq 5 \\
\text { en una escala de 0-10, en las } \\
\text { últimas 24 horas. }\end{array}$ \\
\hline Grado 3 & $\begin{array}{l}\text { Dolor orofaríngeo atribuido a } \\
\text { EAIm, con puntuación media } \geq 6 \\
\text { en una escala de 0-10, en las } \\
\text { últimas 24 horas. }\end{array}$ \\
\hline Grado 0 & $\begin{array}{l}\text { Componente objetivo } \\
\text { ulceración atribuídos a EAIm en } \\
\text { el área orofaríngea). }\end{array}$ \\
\hline Grado 1 & $\begin{array}{l}\text { Eritema oral y/o faríngeo, sin } \\
\text { ulceración, atribuido a EAIm. }\end{array}$ \\
\hline Grado 2 & $\begin{array}{l}\text { Ulceración visible, oral y/o } \\
\text { faríngea, de menos de 7 días de } \\
\text { duración, atribuida a EAIm. }\end{array}$ \\
\hline Grado 3 & $\begin{array}{l}\text { Ulceración visible, oral o } \\
\text { faríngea, con al menos una } \\
\text { lesión de 7 o más días de } \\
\text { duración, atribuida a EAIm. }\end{array}$ \\
\hline
\end{tabular}

Tabla 2. Escala mIAS (mIAS Scale), de valoración de la estomatitis asociada a inhibidores mTOR (EAIm). Adaptado y traducido de Boers-Doets CB y Lalla RV, $2013^{13}$.

\section{PREVENCIÓN}

Dadas las implicaciones que la EAIm puede llegar a tener para los pacientes, la prevención y la detección precoz son de importancia, pues contribuirían a mejorar la calidad de vida del paciente, mantener la ingesta oral y favorecer la continuidad del tratamiento.

Pese a que la aparición de EAIm parece estar asociada a factores como el tipo de tumor, la dosis o el tipo de fármaco utilizado 10,14, sigue siendo necesario aplicar los cuidados preventivos adecuados para reducir su incidencia y gravedad. Estos cuidados coinciden en gran medida con los aplicados en el caso de la estomatitis asociada a la quimioterapia convencional, con alguna excepción.

Los cuidados preventivos están basados en cuatro puntos: (1) la valoración periódica de la boca del paciente, (2) el mantenimiento de un adecuado aporte nutricional, (3) la instauración de una rutina para el cuidado de la mucosa oral, y (4) la educación del paciente en todas estas cuestiones (Tabla 3 ). La valoración de la cavidad oral del paciente es necesaria realizarla de forma regular para lograr la detección precoz y valorar la eficacia de los cuidados preventivos.

Actualmente la evidencia científica relativa a los cuidados de la boca en la prevención de mucositis o EAIm es inadecuada o contradictoria en algunas cuestiones, e inexistente en otras, por lo que las recomendaciones emitidas en las principales guías clínicas están basadas en el consenso de paneles de expertos 12,15. No obstante, dicha evidencia también muestra que la mayoría de los estudios que evalúan protocolos del cuidado de la boca muestran efectos beneficiosos en todas las modalidades de tratamiento oncológico ${ }^{16}$. Es por esto que las recomendaciones actuales se centran principalmente en la inspección diaria de la boca y en el mantenimiento de una adecuada higiene oral (Tabla 3).

Una de las particularidades del cuidado oral en los pacientes en tratamiento con inhibidores mTOR (y con terapias dirigidas en general) es la recomendación de utilizar solución salina para los enjuagues bucales (en los casos de mucositis se recomienda utilizar agua). 
Valoración periódica de la boca

Inspección diaria de la boca por parte del paciente.

Valoración del paciente, preferiblemente con una escala específica para la EAIm (p.ej. escala $m I A S$ ).

Mantener un buen estado nutricional

Mantener un adecuado aporte nutricional durante todo el tratamiento oncológico.

Ingesta de líquidos suficiente para mantener la humedad de la cavidad bucal.

\section{Seguir una rutina de cuidados de la cavidad oral}

Lubricar los labios con crema labial. Hay que tener en cuenta que el uso crónico de vaselina para este fin fomenta la deshidratación de las células de la mucosa y forma una película oclusiva que puede aumentar el riesgo de infecciones.

Hacer higiene dental y de las encías después de cada comida y a la hora de acostarse:

- Utilizar cepillo dental suave, que se renovará mensualmente.

- Si no se tolera el cepillo dental, utilizar bastoncillos específicos para higiene oral.

- Utilizar pasta dental con bajo contenido en flúor y que no forme espuma.

- Cepillar utilizando la técnica de BASS o la técnica de BASS modificada.

- Al finalizar enjuagar la boca minuciosamente con agua.

- Guardar el cepillo en posición vertical, sin tapa, y con las cerdas hacia arriba.

- Si existe el hábito de realizar limpieza interdental (con seda dental o cepillo interdental), puede seguir haciéndose. Si no lo había, no debe comenzar a hacerse durante el tratamiento por el riesgo de romper el epitelio interdental y crear lesiones.

Hacer enjuagues bucales al levantarse y después de cada higiene dental:

- Utilizar unos $15 \mathrm{~mL}$ de un producto sin alcohol, preferiblemente con solución salina.

- Enjuagar y hacer gárgaras durante 1 minuto, aproximadamente.

- En los 30 minutos siguientes evitar comer o beber.

En el caso de utilizar prótesis dental, retirarla antes de realizar el proceso de higiene oral:

- Limpiar las encías.

- Limpiarla la prótesis con cepillo y pasta dental, y aclarar con agua.

- Antes de volver a colocarla, especialmente en el hospital, sumergirla durante 10 minutos en una solución antiséptica (p. ej. clorhexidina al $0,2 \%$ ).

- En el caso de existir algún daño en el epitelio de la mucosa de las encías, evitar poner la prótesis de forma permanente hasta que se haya regenerado.

Evitar la sequedad de la mucosa oral, manteniendo una buena ingesta de líquidos:

- $\quad$ En caso se sequedad recurrente, pueden consumirse caramelos sin azúcar, o masticar chicle sin azúcar.

Evitar acciones o productos que lesionen o deterioren la mucosa oral:

- Por trauma directo:

- Evitar alimentos erosivos (crujientes, con cáscara o espinas, etc...).

- Evitar el uso de seda dental, palillos o cepillos interdentales si no se utilizan habitualmente o si habitualmente su uso provoca sangrado gingival.

- Por irritación
- No fumar
- No consumir alcohol, ni utilizar productos que lo contengan.
- Evitar alimentos ácidos (cítricos, tomate, vinagre,...etc.)
- Evitar alimentos picantes o muy especiados.
O Evitar alimentos muy calientes.

\section{Educación del paciente}

Educar al paciente en las cuestiones abordadas en los tres apartados anteriores, muy especialmente en lo referente al cuidado de la mucosa oral e inspección diaria de la misma.

Tabla 3. Cuidados para la prevención de la estomatitis asociada a inhibidores mTOR (EAIm). Elaborado por los autores, basado en los protocolos y recomendaciones facilitadas en las guías de práctica clínica de la Sociedad Europea de Oncología Médica (ESMO) 12, de la Asociación Multinacional de Cuidados de Soporte en el Cáncer/Sociedad Internacional de Oncología Oral (MASCC/ISOO) 16 y la opinión de varios autores 8,18 .
Pese a que esta recomendación también está basada en opinión de expertos, los mismos consideran que hasta que existan evidencias que sugieran lo contrario, el uso de solución salina es más adecuado.

Los pacientes en tratamiento con terapias dirigidas tienen mayor probabilidad de colonización 
bacteriana y de infecciones, especialmente en el caso de los inhibidores mTOR dado su efecto inmunosupresor ${ }^{17}$. Los enjuagues con solución salina contribuirían a controlar la carga bacteriana de la boca y con ello a prevenir la aparición de EAIm, su progresión, o la aparición de complicaciones infecciosas en las lesiones.

La guía clínica de "Manejo de las lesiones de la mucosa oral y gastrointestinal" de la Sociedad Europea de Oncología Médica (ESMO) propone tanto la solución salina como las soluciones con bicarbonato sódico como adecuadas en la prevención de la EAIm. Ambas recomendaciones lo son en base a opinión de expertos, pero al revisar detenidamente el documento no queda claro si se trata de una doble recomendación o de un error. El uso de enjuagues con soluciones de bicarbonato sódico también ha sido recomendado por otros autores ${ }^{18}$.

\section{MANEJO}

Al igual que ocurre en los cuidados preventivos, las recomendaciones sobre el manejo de la EAIm también están basadas en el consenso de paneles de expertos. Como ya se mencionó anteriormente las lesiones de la EAIm presentan grandes similitudes con las de la estomatitis aftosa, por lo que muchas de las recomendaciones sobre el manejo y el tratamiento son similares ${ }^{19}$. Una vez que aparecen lesiones en la mucosa oral el manejo debe tener como objetivos evitar su progresión, la resolución de las mismas y el tratamiento del dolor (Tabla 4).

La eficacia del uso de corticoides en el tratamiento de las lesiones de la EAIm ha sido mostrada por varios autores, y es una de las principales diferencias con el manejo de la mucositis, donde estos fármacos no han mostrado eficacia y el tratamiento tiene un carácter más paliativo 11,20,21. En la EAIm la tasa de mejoría de las lesiones con el uso tópico, sistémico 0 intralesional de corticoides llega a ser de hasta el $87 \%{ }^{8}$.

En los casos leves y moderados la vía tópica debe considerarse como primera elección, utilizando enjuagues o la aplicación directa en gel, dependiendo del número de lesiones a tratar y la accesibilidad de las mismas. El uso sistémico de corticoides a dosis altas se reserva para casos graves o recurrentes de EAIm, o para aquellos en los que las lesiones afectan también al esófago. En dichos casos, especialmente en aquellos con grado 3 en los dos componentes de la escala mIAS (Tabla 1) y en los que el manejo no es efectivo en la resolución de las lesiones o en el control del dolor, puede ser necesario una reducción de dosis en el tratamiento con inhibidores mTOR, e incluso su interrupción temporal hasta que las lesiones mejoren a grado I o desaparezcan 22-24. En muchos casos, tras dicha reducción de dosis o interrupción del tratamiento, las lesiones se resuelven y no reaparecen tras reiniciar el tratamiento ${ }^{8}$.

En el caso de úlceras persistentes que no se resuelven, de gran tamaño y/o muy dolorosas, suele ser conveniente la valoración de un experto que considere la inyección intralesional de corticoides (p.ej. una dosis semanal de triamcinolona). 
Esta medida parece mostrar una mejoría inmediata de los síntomas ${ }^{8}$.
El manejo del dolor es un aspecto muy importante para mejorar la calidad de vida del paciente $y$ preservar la ingesta oral.

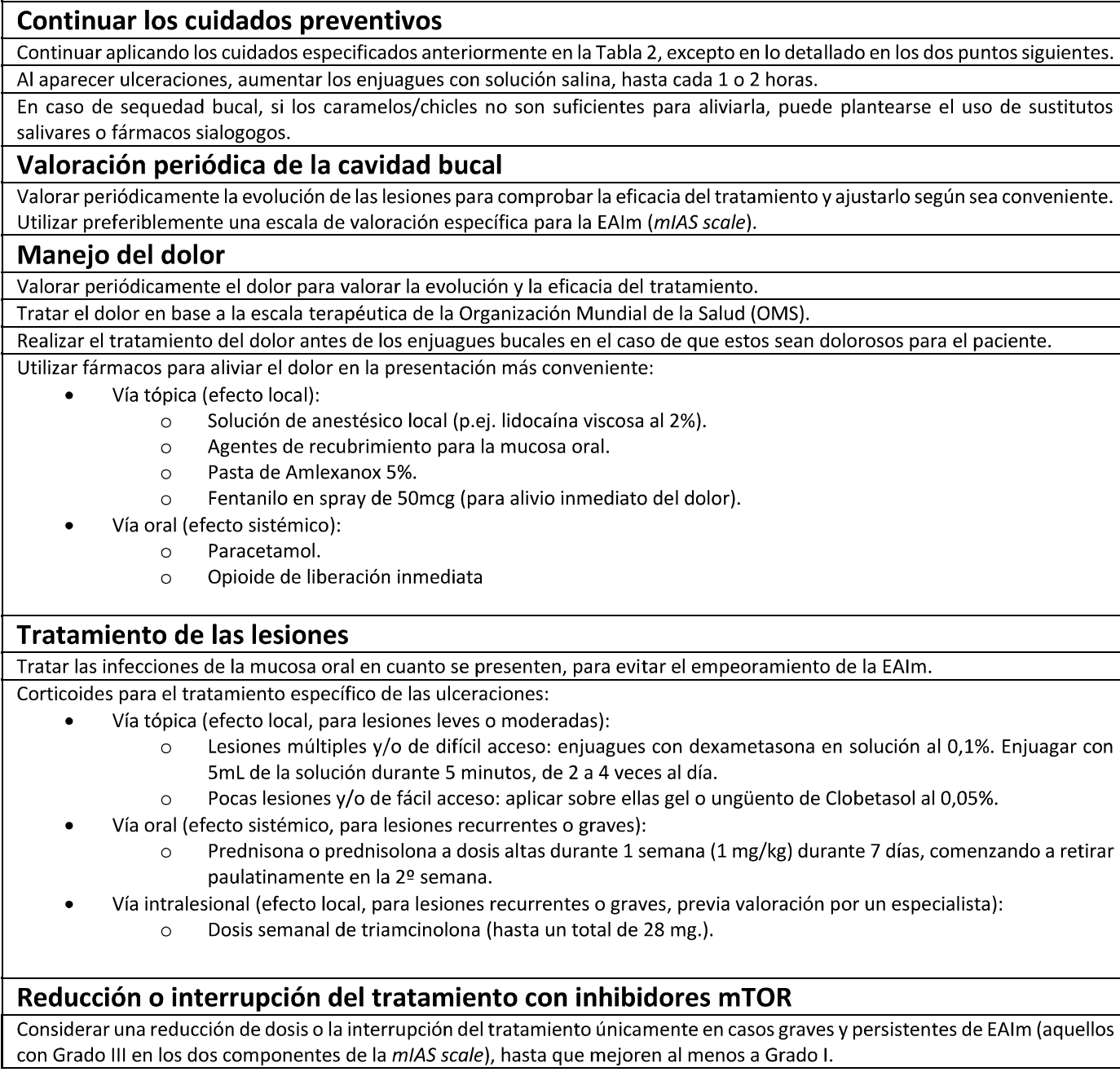

- Prednisona o prednisolona a dosis altas durante 1 semana $(1 \mathrm{mg} / \mathrm{kg})$ durante 7 días, comenzando a retirar paulatinamente en la 20 semana.

- Vía intralesional (efecto local, para lesiones recurrentes o graves, previa valoración por un especialista):

- Dosis semanal de triamcinolona (hasta un total de $28 \mathrm{mg}$.).

\section{Reducción o interrupción del tratamiento con inhibidores mTOR}

Considerar una reducción de dosis o la interrupción del tratamiento únicamente en casos graves y persistentes de EAIm (aquellos con Grado III en los dos componentes de la mIAS scale), hasta que mejoren al menos a Grado I.

Tabla 4. Manejo de la estomatitis asociada a inhibidores mTOR (EAIm). Válido en cualquier tipo de cáncer y tipo de terapia dirigida, incluidos los inhibidores mTOR. Elaborado por los autores, basado en los protocolos y recomendaciones facilitadas en las guías de práctica clínica de la Sociedad Europea de Oncología Médica (ESMO) 12, de la Asociación Multinacional de Cuidados de Soporte en el Cáncer/Sociedad Internacional de Oncología Oral (MASCC/ISOO) 16 y la opinión de varios autores 3,8,18,19,22.
Es conveniente realizar una valoración periódica del dolor o de la sensibilidad bucal con el objeto de valorar la evolución de la EAIm, la eficacia del tratamiento analgésico y el ajuste de las dosis y fármacos utilizados. Las recomendaciones aconsejan seguir un esquema de manejo del dolor basado en las recomendaciones de la OMS, utilizando analgésicos por vía tópica $y / u$ oral, en función de las necesidades de cada paciente. En el caso de que los enjuagues 
resulten dolorosos, es necesario aplicar con antelación el tratamiento analgésico con objeto de que la rutina diaria del cuidado de la boca pueda realizarse correctamente.

\section{DISCUSIóN}

En este artículo se ha revisado las particularidades de la EAIm, basándose en la evidencia disponible y en las recomendaciones facilitadas por las principales guías clínicas y otros artículos de revisión. No obstante, debido a la relativa novedad en el uso de inhibidores mTOR aún no existe un cuerpo de conocimiento importante sobre la valoración, prevención y manejo de la EAIM que hace que las recomendaciones existentes estén basadas únicamente en consenso de expertos. Esto tiene implicaciones muy importantes para la investigación.

Por ejemplo, pese a que existe una escala específica para su valoración (mIAS Scale) ésta no cuenta con estudios de validación, y por lo tanto no se conocen los valores de validez (sensibilidad y especificidad) y fiabilidad de dicha escala. Tampoco se ha hecho la adaptación transcultural en castellano, que habilite convenientemente su uso en nuestro entorno. Sería conveniente avanzar en este sentido.

Las cifras de incidencia de la EAIm presentes en la literatura podrían ser imprecisas, ya que muchos de los estudios que aportan este tipo de dato no han sido diseñados de forma específica para este fin, sino para analizar datos generales de seguridad y eficacia del tratamiento con inhibidores mTOR. Además, el uso de diversas escalas para su valoración dificulta la comparabilidad de los datos. El diseño de estudios especialmente orientados a estimar la incidencia de la EAIm utilizando una herramienta de valoración específica podría mejorar la precisión de la estimación de la incidencia de EAIm.

La frecuencia y la gravedad de la EAIm parece estar asociada a ciertas variables, pero los estudios sobre los factores asociados a su desarrollo aún son escasos. Conocer los factores de riesgo, especialmente si son modificables, permitiría mejorar la estrategia de prevención.

Por último, conocer qué producto es más adecuado para el cuidado de la boca es otro aspecto que aún no cuenta con una evidencia clara. $Y$ por ello las estrategias actuales en la prevención y manejo de la EAIm están basadas en el consenso de expertos. Aunque parecen funcionar razonablemente bien, sería necesario el desarrollo de ensayos clínicos bien diseñados para evaluar la eficacia de cada producto, sus ventajas e inconvenientes.

En conclusión, la EAIm es un evento adverso que puede ser confundido con la mucositis asociada a la quimioterapia convencional. Ambas son procesos diferentes, que requieren abordajes diferentes. El manejo adecuado de la EAIm propiciaría una mejora de la calidad de vida del paciente, la resolución temprana de las lesiones y la continuidad del tratamiento. No obstante, la EAIm no cuenta con un cuerpo de conocimiento importante respecto a su valoración, prevención y manejo, lo que tiene implicaciones 
importantes para la investigación en los próximos años.

\section{REFERENCIAS}

1. Martins $F$, de Oliverira $M A$, Wang $Q$, Sonis S, Gallotini M, George S, Treister N. A review of oral toxicity associated with mTOR inhibitor therapy in cancer patients. Oral Oncol. 2013; 49(4): 293-8. DOI: 10.1016/j.oraloncology.2012.11.008

2. Watters AL, Epstein JB, Agulnik M. Oral complications of targeted cancer therapies: a narrative literature review. Oral Oncol. 2011; 47(6): 441-8. DOI: 10.1016/j.oraloncology.2011.03.028

3. Boers-Doets CB, Raber-Durlacher JE, Treister NS, et al. Mammalian target of rapamycin inhibitor-associated stomatitis. Future Oncol. 2013; 9: 1883-92. DOI: 10.2217/fon.13.141

4. Li E, Trovato JA. New developments in management of oral mucositis in patients with head and neck cancer or receiving targeted anticancer therapies. Am J Health Syst Pharm. 2012; 69(12): 1031-7. DOI: 10.2146/ajhp100531

5. Sonis ST. Oral mucositis. Anticancer Drugs. 2011; 22(7): 607-12. DOI: 10.1097/CAD.0b013e3283462086

6. Scully C. Aphthosus ulceration. N Engl J Med. 2006; 355(2): 165-72. DOI: 10.1056/NEJMcp054630

7. Sonis S, Treister N, Chawla S, Demetri G, Haluska F. Preliminary characterization of oral lesions associated with inhibitors of mammaliam target of rapamycin in cancer patients. Cancer. 2010; 116(1): 210-5. DOI: $10.1002 / \mathrm{cncr} .24696$

8. de Oliveira MA, Martins e Martins $F$, Wang Q, Sonis S, Demetri G, George S, Butrynski J, Treister NS. Clinical presentation and management of mTOR inhibitor-associated stomatitis. Oral Oncol. 2011; 47(10): 998-1003. DOI: 10.1016/j.oraloncology.2011.08.009 9. Ferté C, Paci A, Zizi M, Gonzales DB, Goubar A, Gomez-Roca C, Massard C, Sahmoud T, André F, Soria JC. Natural history, management and pharmacokinetics of everolimus-inducedoral ulcers: insights into compliance issues. Eur J Cancer 2011; 47(15): 224955. DOI: $10.1016 / j$. ejca.2011.03.017 10. Shameem R, Lacouture M, Wu S. Incidence and risk of high-grade stomatitis with mTOR inhibitors in cancer patients. Cancer Invest. 2015; 33(3): 70-7. DOI: 10.3109/07357907.2014.1001893

11. Kalogirou EM, Tosios KI, Piperi EP, Sklavounou A. mTOR inhibitor-associated stomatitis (mIAS) in three patients with cancer treated with everolimus. Oral Surg Oral Med Oral Pathol Oral Radiol. 2015; 119(1): e13-9. DOI: 10.1016/j.0000.2014.08.023

12. Peterson DE, Boers-Doets CB, Bensadoun RJ, Herrstedt J, ESMO Guidelines Committee. Management of oral and gastrointestinal mucosal injury: ESMO Clinical Practice Guidelines for diagnosis, treatment, and follow-up. Ann Oncol. 2015; Suppl 5: v139-51. DOI: 10.1093/annonc/mdv202

13. Boers-Doets CB, Lalla RV. The mIAS scale: a scale to measure mTOR inhibitorassociated stomatitis. Support Care Cancer. 2013; 21 (Suppl 1): S140. DOI: 10.1007/s00520-013-1798-3

14. Lo Muzio L, Arena C, Troiano G, Villa A. Oral stomatitis and mTOR inhibitors: a review of current evidence in 20,915 patients. Oral Dis. 2018; 24(1-2): 144-71. DOI: $10.1111 /$ odi.12795

15. Lalla RV, Bowen J, Barasch A, Elting L, Epstein J, Keefe DM, McGuire DB, Migliorati C, Nicolatou-Galitis O, Peterson DE, Raber-Durlacher JE, Sonis ST, Elad S. Mucositis Guidelines Leadership Group of the Multinational Association of Supportive Care in Cancer and International Society of Oral Oncology (MASCC/ISOO). MASCC/ISOO clinical practice guidelines for the management of mucositis secondary to cancer therapy. Cancer. 2014; 120(10): 1453-61. DOI: $10.1002 / \mathrm{cncr} .28592$

16. McGuire DB, Fulton JS, Park J, Brown CG, Correa ME, Eilers J, Elad S, Gibson F, Oberle-Edwards LK, Bowen J, Lalla RV; Mucositis Study Group of the Multinational Association of Supportive Care in Cancer/International Society of Oral Oncology (MASCC/ISOO). Systematic review of basic oral care for the management of oral mucositis in cancer patients. Support Care Cancer. 2013; 21(11): 3167-77. DOI: $10.1007 / \mathrm{s} 00520-$ 013-1942-0

17. Malizzia LJ, Hsu A. Temsirolimus, an mTOR inhibitor for treatment of patients with advanced renal cell carcinoma. Clin J Oncol Nurs. 2008; 12: 639-46. DOI: 10.1188/08.CJON.639-646

18. Pilotte AP, Hohos MB, Polson KM, Huftalen TM, Treister N. Managing stomatitis in patients treated with mammalian target of rapamycin inhibitors. Clin J Oncol Nurs. 2011; 15(5): E83-9. DOI: 10.1188/11.CJON.E83-E89

19. Peterson $E, O^{\prime}$ Shaughnessy JA, Rugo HS, Elad S, Schubert MM, Viet CT, Campbell-Baird C, Hronek J, Seery V, 
Divers J, Glaspy J, Schmidt BL, Meiller TF. Oral mucosa injury caused by mammalian target of rapamycin inhibitors: emerging perspectives on pathobiology and impact on clinical practice. Cancer Med. 2016; 5(8): 1897-907. DOI: $10.1002 /$ cam4.761 20. Chuang $P$, Langone AJ. Clobetasol ameliorates aphthosus ulceration in renal transplant patients on sirolimus. Am J Transplant. 2007; 7(3): 714-7. DOI: 10.1111/j.1600-6143.2006.01678.x

21. Meiller TF, Varlotta S, Weikel D. Recognition and management of oral mucosal injury caused by mammalian target of rapamycin inhibitors: a case series. Case Rep Oncol. 2015; 8(2): 36977. DOI: $10.1159 / 000438747$

22. Dy GK, Adjei AA. Understanding, recognizing, and managing toxicities of targeted anticancer therapies. CA Cancer ] Clin. 2013; 63(4): 249-79. DOI: 10.3322/caac. 21184

23. Aapro M, Andre F, Blackwell K, Calvo E, Jahanzeb $M$, Papazisis K, Porta C, Pritchard K, Ravaud A. Adverse event management in patients with advanced cancer receiving oral everolimus: focus on breast cancer. Ann Oncol. 2014; 25(4): 763-73. DOI: 10.1093/annonc/mdu021 24. Porta C, Osanto S, Ravaud A, Climent MA, Vaishampayan U, White DA, Creel $P$, Dickow B, Fischer P, Gornell SS, Meloni F, Motzer RJ. Management of adverse events associated with the use of everolimus in patients with advanced renal cell carcinoma. Eur J Cancer. 2011; 47(9): 1287-98.

DOI: 